\title{
Microsurgical scleral drainage and trabeculectomy-scleral flap adjustable suture combination technique in the treatment of primary glaucoma
}

\author{
Li Xiahou', Chunlan Liu'², \\ Weihong Zhou ${ }^{3}$, Shasha Yang ${ }^{4}$
}

\begin{abstract}
Objective: To investigate the clinical effect of microsurgical scleral drainage and trabeculectomy combined with scleral flap adjustable suture technique in the treatment of primary glaucoma.

Methods: One hundred primary glaucoma patients (120 eyes) in Xinyu People's Hospital of Jiangxi province were selected from July 2014 to June 2016. The patients were randomly divided into control group and study group. The control group was treated with compound trabeculectomy, while the study group was treated with microsurgical scleral drainage and trabeculectomy combined with scleral flap adjustable suture technique. In both groups of patients, intraocular pressure, functional filtering bleb formation, and complications before and after surgery were monitored for three days, one week, one month, three months, six months and one year, while anterior chamber depth was determined one week after operation. The extent of success of operation was compared between the two groups.

Results: At three days, one week, one month, three months, six months and one year after surgery, intraocular pressure of study group was significantly lower than that of the control group $(P<0.05)$. There was $93.33 \%$ formation of functional filtering blebs in the study group, which was significantly higher than that in the control group $(60.00 \%, \mathrm{P}<0.001)$. Moreover, normal anterior chamber formation was significantly higher in the study group (91.67\%) than in the control group $(71.67 \%, \mathrm{P}<0.01)$. There was $95.00 \%$ operation success in the study group, relative to $68.33 \%$ success in the control group $(P<0.001)$.

Conclusion: Microsurgical scleral drainage and trabeculectomy combined with scleral flap adjustable suture technique has better curative effect on primary glaucoma than compound trabeculectomy. Moreover, it does not exacerbate complications. Therefore, the combination treatment technique merits clinical application.
\end{abstract}

KEYWORDS: Primary glaucoma, Trabeculectomy, Scleral flap adjustable suture, Scleral space drainage pool.

doi: https://doi.org/10.12669/pjms.36.2.1439

How to cite this:

Xiahou L, Liu C, Zhou W, Yang S. Microsurgical scleral drainage and trabeculectomy-scleral flap adjustable suture combination technique in the treatment of primary glaucoma. Pak J Med Sci. 2020;36(2):234-239. doi: https://doi.org/10.12669/pjms.36.2.1439

This is an Open Access article distributed under the terms of the Creative Commons Attribution License (http://creativecommons.org/licenses/by/3.0), which permits unrestricted use, distribution, and reproduction in any medium, provided the original work is properly cited.

Correspondence:

Shasha Yang,

No.1 Panfu Road,

The Second Affiliated Hospital,

South China University of Technology,

Guangzhou First People's Hospital,

Guangzhou, 510180, China.

E-mail: hlxhoulixia@163.com

* Received for Publication:

* $1^{\text {st }}$ Revision Received:

* $2^{\text {nd }}$ Revision Received:

* Final Revision Accepted:
July 30, 2019

October 31, 2019

November 15, 2019

November 19, 2019

\section{INTRODUCTION}

Glaucoma is an eye disease with clinical characteristics of optic nerve degeneration and visual field defect, which are not readily detected, but are progressive and irreversible; $30 \%$ of people with glaucoma become blinded., Visual function in glaucoma patients is severely damaged, usually manifesting as optic atrophy and visual field defect which are serious threats to the visual function and 
daily lives of patients. ${ }^{3}$ Available clinical data show that the incidence of glaucoma in China is $20 \%$ $40 \%{ }^{4}$ Therefore, the development of newer and more effective treatment strategies for glaucoma is of immense clinical significance. Surgical treatment is the main method of treating primary glaucoma. Surgical treatment effectively reduces intraocular pressure and protects and saves visual function. ${ }^{5}$ Trabeculectomy, the standard therapy for glaucoma, has a beneficial effect of lowering intraocular pressure. ${ }^{6,7}$ However, clinical practice has revealed that in the course of treatment, patients' filters are susceptible to tissue edema, ${ }^{8}$ fibroplasia and wound repair, which may result in blockage of filters, interruption of drainage, and ultimately operation failure. Moreover, the incidence of operationrelated complications may be high, resulting in poor therapeutic outcomes. Trabeculectomy combined with scleral flap adjustable suture technique is an improvement on the trabeculectomy technique. ${ }^{9}$ The application of scleral flap adjustable suture technique forms anterior chamber early, to reduce the incidence of shallow anterior chamber and choroidal detachment. Moreover, it achieves quantitative adjustment of aqueous humor filtration volume by controlling the time of suture removal and number of removed sutures, thereby forming ideal functional filtration blebs, leading to ideal intraocular pressure control, although connective tissue proliferation can still lead to surgery failure. ${ }^{10}$ In recent years, it has been reported that trabeculectomy combined with sclera pool not only establishes a lasting outflow channel of aqueous humor and solves the problem of early excessive filtering after surgery, but also prevents scleral flaps from adhering to the scleral bed, thereby effectively preventing scar formation. ${ }^{11}$

The purpose of this study was to investigate the clinical effect of microsurgical scleral drainage and trabeculectomy combined with scleral flap adjustable suture technique in the treatment of primary glaucoma, with a view to providing reference for clinical practice.

\section{METHODS}

One hundred patients (120 eyes) with primary glaucoma who were hospitalized in Xinyu People's Hospital of Jiangxi province from July 2014 to June 2016 were selected. All patients met the diagnostic criteria of primary glaucoma. ${ }^{12}$ They were divided into control group and study group, using the random number table. There were 52 cases (60 eyes) in the study group, and 48 cases (60 eyes) in the control group. In all, there were 62 cases of acute primary angle closure glaucoma (APACG) (68 eyes), 28 cases of chronic primary angle closure glaucoma (CPACG) (36 eyes), and 10 cases of primary open angle glaucoma (POAG) (16 eyes). Patients in the study group were treated with microsurgical scleral drainage and trabeculectomy combined with scleral flap adjustable suture technique. In contrast, compound trabeculectomy was performed in the control group. The angle of anterior chamber was re-examined before operation. The dynamic angle of the chamber was still lower than 180 degrees after lowering of intraocular pressure and pupil contraction. There were no significant differences in sex, age, baseline intraocular pressure and type of disease between the two groups ( $\mathrm{P}>0.05$, Table-I).

Inclusion criteria: Diagnosis of APACG, CPACG and POAG consistent laid down creteria, ${ }^{13}$ and provision of informed consent by the patient for operation after being duly informed about relevant treatments and surgical scheme.

Exclusion criteria: Patients who previously underwent trabecular surgery twice, and patients who underwent combined surgery were excluded. Moreover, patients with refractory glaucoma, secondary glaucoma, developmental glaucoma, severe cardiovascular diseases or other severe organic diseases, severe diseases in the immune system, as well as those with a history of psychiatric or nervous system disease, were also excluded. This study was reviewed and approved by the medical ethical committee (Dated December 11, 2018) of the hospital.

Surgical methods: All operations in the control group and study group were carried out by the same surgical team with bedside clinicians as surgical assistants. The operation procedures in the study group were as follows: After conventional disinfection and spreading of aseptic towel, surface and subconjunctival anesthesia were performed. The eyelid was opened using an eye speculum. The superior rectus was fixed. High-position conjunctival flap which took corneal limbus as the fundus, was made at the site which was $8-10 \mathrm{~mm}$ above corneal limbus in the direction of 12 o'clock position. The Tenon's capsule was separated to expose an $8 \times 7 \mathrm{~mm}$ sclera surgical field. The blood vessels on the surface of the sclera were burned to stop bleeding. Then, a $5 \mathrm{~mm} \times 6 \mathrm{~mm}$ scleral flap which took the corneal limbus as the fundus, and was one-third the thickness of the sclera, was made. A sclera tunnel knife was used to separate forward to the site which was one $\mathrm{mm}$ inside transparent 
cornea. Then, a $3 \times 3 \mathrm{~mm}$ middle layer scleral flap of thickness one-third that of the sclera was removed, taking the corneal limbus as the fundus in the middle below the shallow scleral flap.

A $4 \mathrm{~mm} \times 3.5 \mathrm{~mm}$ cotton fleexe immersed in 0.25 $0.33 \mathrm{mg} / \mathrm{mL}$ mitomycin $\mathrm{C}$ was placed below the scleral flap and conjunctival flap for 2-3 minutes. Then, the surgical field and cornea were washed with $150 \mathrm{~mL}$ of BBS. An anterior chamber puncture was made on the corneal limbus at the 9 o'clock position using a paracentesis knife. Aqueous fluid was slowly released via the puncture mouth so as to further reduce theintraocular pressure. Then, $1.5 \times 1.5$ $\mathrm{mm}$ trabecular meshwork and deep corneosclera tissue below the scleral flap were removed along the posterior margin of sclera ridge. This was followed with peripheral iridectomy. The top of each side of the scleral flap was sutured (one stitch) with 10/0 non-absorbable nylon thread. A small amount of BBS was injected via the puncture mouth of the anterior chamber to reconstruct the anterior chamber, and some aqueous fluid was discharged below the scleral flap. Then, two adjustable sutures were made at the bottom of the scleral flap. The degree of tightness was adjusted, and the tightness was suitable when the seepage below the scleral flap slowly emerged to maintain the depth of the anterior chamber, or when the intraocular pressure was normal or low. Tenon's capsule was sutured (2-3 stitches) using 10-0 non-absorbable nylon thread. The incision on the bulbar conjunctiva was continuously sutured using 10-0 non-absorbable nylon thread. Dexamethasone $(5 \mathrm{mg}$ ) was injected subconjunctivally, and erythromycin eye ointment was used. The operated eye was bound up. After surgery (usually 3-14 days), the two adjustable sutures were removed, depending on the value of intraocular pressure and chamber pressure.

Compound trabeculectomy was used in the control group. The operation was performed under a microscope. Surface and subconjunctival anesthesia were performed. The eyelid was opened using an eye speculum. The superior rectus was fixed. The Tenon's capsule was separated to expose a $8 \times 7 \mathrm{~mm}$ sclera surgical field. Then, a $4 \mathrm{~mm} \times 5 \mathrm{~mm}$ scleral flap which took the corneal limbus as the fundus, and was one-third of sclera thickness, was made. A sclera tunnel knife was used to separate forward to the site which was one $\mathrm{mm}$ inside transparent cornea. A $4 \mathrm{~mm} \times 3.5 \mathrm{~mm}$ cotton fleexe immersed in $0.25-0.33 \mathrm{mg} / \mathrm{mL}$ mitomycin $\mathrm{C}$ was placed below the scleral flap and conjunctival flap for 2-3 minutes. Then, the surgical field and cornea were washed with $150 \mathrm{~mL}$ of BBS. An anterior chamber puncture was performed using a paracentesis knife to slowly release aqueous fluid to achieve slow decrease of intraocular pressure. Trabecular meshwork and deep corneosclera tissue below the scleral flap were removed along the posterior margin of sclera ridge. Then, peripheral iridectomy was performed. The top and bottom on both sides of the scleral flap were sutured (one stitch). The conjunctival incision was continuously sutured, and $5 \mathrm{mg}$ of dexamethasone was subconjunctivally injected. Erythromycin eye ointment was applied, and the operative eye was bound up.

Clinical indicators: The indices determined were intraocular pressure and formation of functional filter-bubble before surgery, and three days, one week, one month, three months, six months and one year after surgery. Moreover, the depth of anterior chamber at one week after operation was determined.

Statistical method: Statistical analysis was performed using SPSS 17.0. Measurement data are expressed as mean \pm standard deviation. Two-group comparisons were done using t-test. Enumeration data are expressed as percentage. Statistical differences in enumeration data were compared between the two groups using Chi-square test. Differences were considered statistically significant at $\mathrm{P}<0.05$.

Table-I: General data of the study group and the control group.

\begin{tabular}{|c|c|c|c|c|c|}
\hline \multicolumn{2}{|l|}{ Group } & Study group & Control group & $X^{2} / t$ value & $P$ value \\
\hline \multicolumn{2}{|l|}{ Patients (n) } & 52 & 48 & / & / \\
\hline \multicolumn{2}{|l|}{ Eyes (n) } & 60 & 60 & / & / \\
\hline \multicolumn{2}{|c|}{ Male/Female (n/n) } & $21 / 31$ & $20 / 28$ & 0.017 & $>0.05$ \\
\hline \multirow[t]{3}{*}{ Glaucoma type } & APACG & 35 & 33 & 0.003 & $>0.05$ \\
\hline & CPACG & 17 & 19 & & \\
\hline & POAG & 8 & 8 & & \\
\hline \multicolumn{2}{|l|}{ Ages } & $58.13 \pm 13.23(45-79)$ & $57.59 \pm 11.82(43-81)$ & 0.235 & $>0.05$ \\
\hline \multicolumn{2}{|c|}{ Intraocular pressure $(\mathrm{mmHg})$} & $50.51 \pm 5.91$ & $49.82 \pm 6.25$ & 0.621 & $>0.05$ \\
\hline
\end{tabular}


Table-II: Intraocular pressure at different time periods before and after the operation in the study group and the control group ( $\mathrm{mmHg}$ ).

\begin{tabular}{lcccc}
\hline Group & Study group & Control group & t value & P value \\
\hline Before & $50.51 \pm 5.91$ & $49.82 \pm 6.25$ & 0.621 & $>0.05$ \\
After three days & $11.41 \pm 2.37$ & $13.15 \pm 2.82$ & 3.659 & $<0.05$ \\
After one week & $12.86 \pm 2.05$ & $14.30 \pm 2.87$ & 2.943 & $<0.05$ \\
After one month & $12.42 \pm 2.18$ & $15.61 \pm 3.54$ & 5.944 & $<0.05$ \\
After three months & $12.18 \pm 2.53$ & $17.44 \pm 4.46$ & 7.946 & $<0.05$ \\
After six months & $12.36 \pm 2.64$ & $20.73 \pm 5.81$ & 10.159 & $<0.05$ \\
After twelve months & $13.47 \pm 4.12$ & $21.88 \pm 6.26$ & 8.962 & $<0.05$ \\
\hline
\end{tabular}

Table-III: Comparison of filtering blebs at one year after operation.

\begin{tabular}{lcccc}
\hline Group & \multicolumn{2}{c}{ Functional filtering blebs } & \multicolumn{2}{c}{ Non-functional filtering blebs } \\
\hline & I-type & II-type & III-type & VI-type \\
\hline Study group & $11(18.33 \%)$ & $45(75.00 \%)$ & $3(5.00 \%)$ & $1(1.67 \%)$ \\
Control group & $9(15.00 \%)$ & $27(45.00 \%)$ & $17(28.33 \%)$ & $7(11.67 \%)$ \\
$X^{2}$ value & & & 18.634 & \\
P value & & $<0.001$ & & \\
\hline
\end{tabular}

\section{RESULTS}

There was no significant difference in the preoperative intraocular pressure value between the study group and control group $(\mathrm{P}>0.05)$. However, the intraocular pressure of the study group was significantly lower than that of the control group $(\mathrm{P}<0.05$, Table-II).

The extent of formation of functional filtering blebs in the study group was significantly higher than that in the control group $(\mathrm{P}<0.001)$. Table-III.

One week after the operation, the extent of formation of normal anterior chamber in the study group was significantly higher than that in the control group $(\mathrm{P}<0.01$, Table-IV). There were no significant differences in the incidence of postoperative complications between the two groups ( $\mathrm{P}>0.05$, Table-V).

In the study group, operation was successful in 57 eyes $(95.00 \%)$ comprising 54 eyes which were completely successful $(90.00 \%)$ and 3 eyes which were conditionally successful $(5.00 \%)$. Three eyes failed $(5.00 \%)$. In the control group, operation was successful in 41 eyes (68.33\%), comprising 34 eyes which were completely successful (56.67\%) and 7 eyes which were conditionally successful $(11.67 \%)$; 19 eyes failed $(31.67 \%)$. The success of operation in the study group was significantly higher than that in the control group $\left(X^{2}=14.249, \mathrm{P}<0.001\right)$.

\section{DISCUSSION}

Primary glaucoma is divided into PACG and POAG, based on differences in pathogenesis and anatomical structure of angle of chamber. Moreover, depending on degree of onset and clinical course, PACG is sub-divided into APACG and CPACG. ${ }^{14-16}$ It has been reported that PACG is the most common form of glaucoma in China. ${ }^{17}$ The

Table-IV: Comparison of the onset of anterior chamber in the study group and the control group one week after the operation.

\begin{tabular}{lcccc}
\hline Group & Study group & Control group & $X^{2}$ value & P value \\
\hline Normal & $55(91.67 \%)$ & $43(71.67 \%)$ & 8.015 & 0.005 \\
I-grade & $5(8.33 \%)$ & $14(23.33 \%)$ & & \\
II-grade & $0(0 \%)$ & $3(5.00 \%)$ & & \\
III-grade & $0(0 \%)$ & $0(0 \%)$ & & \\
\hline
\end{tabular}

Table-V: Comparison of incidence of complications between the study group and control group.

\begin{tabular}{lcccc}
\hline Group & Study group & Control group & $X^{2}$ value & P value \\
\hline Postoperative corneal edema & $7(11.67 \%)$ & $6(10.00 \%)$ & 1.174 & 0.965 \\
Fibrinous exudate in pupillary area and anterior & $3(5.00 \%)$ & $2(3.33 \%)$ & & \\
$\quad$ chamber inflammation & $2(3.33 \%)$ & $3(5.00 \%)$ & & \\
Postoperative low intraocular pressure & $1(1.67 \%)$ & $0(0 \%)$ & & \\
Filtering bleb leak & $0(0 \%)$ & $2(3.33 \%)$ & $/$ & $/$ \\
Malignant glaucoma &
\end{tabular}


control of intraocular pressure is the only effective method of treating glaucoma at present. ${ }^{18}$ Filtering surgery is one of the methods for glaucoma treatment. This entails the creation of an artificial drainage for aqueous humor through surgery so as to reduce intraocular pressure.

Trabeculectomy is the traditional and the most common and classical surgical method for glaucoma treatment. Through this operation, aqueous humor can be drained to the subconjunctival space and absorbed to form follicles. ${ }^{19}$ The high degree of failure in trabeculectomy $(20 \%-30 \%)$, limits its application. ${ }^{20}$ Fibrous hyperplasia, tissue edema and wound repair are the causes of failure of trabeculectomy. When the filters are blocked, drainage is interrupted. Then, the filters become adhesive, leading to difficultly in formation of functional follicles. ${ }^{21}$ A clinical study found that mitomycin $C$ adjuvant therapy with trabeculectomy had a significant effect. Mitomycin $C$ is an antineoplastic antibiotic derived from Streptomyces cephala. ${ }^{22}$ It significantly inhibits the proliferation of fibroblasts and reduces the formation of scars. Its pharmacological effect is significant. Mitomycin C adjuvant therapy effectively alleviates the scarring of filter passage after operation, although it can also result in the occurrence of shallow anterior chamber post-surgery. Shallow anterior chamber causes serious consequences such as bullous keratopathy, iris adhesion, malignant glaucoma, cataracts, choroidal hemorrhage and detachment due to damaged corneal endothelial cells, leading to failure of operation and abnormal intraocular tissue structure. The use of adjustable suture for scleral flaps solves the problem of shallow anterior chamber caused by over filtration in the early stage, resulting in significant, reduction of post-surgery complications and improvement in degree of operation success. However, in the long run, intraocular pressure may increase again due to the proliferation of connective tissue at the scleral opening at the angle of the chamber, and the inhibition of aqueous humor outflow induced by the healing of scleral lamellar wound. These can lead to failure of operation. In recent years, it has been found that trabeculectomy combined with scleral pool effectively establishes a lasting outflow channel for aqueous humor, reduces intraocular pressure, prevents scleral flap from adhering to scleral bed, and reduces scarring. ${ }^{23}$ Therefore, trabeculectomy was combined with adjustable sutured flap, in order to artificially control the depth of anterior chamber and intraocular pressure after trabeculectomy, and improve the success of surgery.

The results of this study showed that trabeculectomy in combination with adjustable sutured flap effectively reduced intraocular pressure in short term and long term, and promoted the formation of functional filtering blebs, when compared with compound trabeculectomy. It might be due to enhanced discharge of aqueous humor from the conjunctiva, and also through uveosceleral outflow in the short-term study group. Moreover, the intraocular pressure was maintained at a normal level for a long time in the study group because of the formation of filtration channel scar. The incidence of shallow anterior chamber in the study group was low, probably because adjustable sutures artificially control the filtration volume, and adjusting the suture tightness controlled the outflow resistance of aqueous humor after passing through the dangerous shallow anterior chamber. Results of the follow-up showed that operation success in the study group was significantly higher than that in the control group, which was in line with expectations.

\section{CONCLUSION}

The application of microsurgical scleral drainage and trabeculectomy in combination with scleral flap adjustable suture technique in primary glaucoma effectively controls early filtration flow and speed of aqueous humor, and reduces the incidence of low intraocular pressure and shallow anterior chamber caused by strong filtration. Moreover, it reduces scars, promotes the formation of functional filtering blebs and the establishment of permanent aqueous humor filtration channels, and improves the success of glaucoma surgery. Therefore, this combination technique merits clinical application and promotion.

Acknowledgement: This study was supported by the project of science and technology plan of Jiangxi under grant number 20142BBG70040.

Declaration of interest: All authors declared there was no conflict interests involved.

\section{Grant Support \& Financial Disclosures: None.}

\section{REFERENCES}

1. Garcia-Martin E, Pablo L, Ferreras A, Idoipe M, Perez S, Pueyo V. Valor predictivo del Heidelberg Retina Tomograph III en pacientes con glaucoma incipiente o sospecha de glaucoma. Archivos de la Sociedad Espanola de Oftalmologia. 2010;85(4):138-143. 
2. Suarez-Fernandez MJ, Gutierrez-Diaz E, Julve San Martin A, Fernandez-Reyes MF, Mencía-Gutierrez E. Vitrectomia via pars plana e implante simultaneo de dispositivo de drenaje para glaucoma. Archivos de la Sociedad Espanola de Oftalmologia. 2010;85(3):97-102.

3. El-Saied HM, Foad PH, Eldaly MA, Abdelhakim MA. Surgically induced astigmatism following glaucoma surgery in Egyptian patients. J Glaucoma. 2014;23(3):190193. doi: $10.1097 /$ IJG.0000000000000035

4. Kaplan A, Kocaturk T, Dayanir V. The effect of adjustable suture (Khaw) trabeculectomy on intraocular pres-sure: a retrospective case series. Int Ophthalmol. 2016;36(1):97-104. doi: 10.1007/s10792-015-0086-2

5. Tian LJ, Li M. Clinical observation of improved trabeculectomy in glaucoma. J Injuries Occupat Dis Eye Ophthalmic Surg. 2010;32(9):693-694. doi: 10.3760/cma.j.is sn.2095-1477.2010.09.020

6. Delbeke H, Stalmans I, Vandewalle E, Zeyen T. The effect of trabeculectomy on astigmatism. J Glaucoma. 2016;25(4):e308-e312. doi: 10.1097/IJG.0000000000000236

7. Marco S, Damji KF, Nazarali S, Rudnisky CJ. Cataract and glaucoma surgery: Endoscopic cyclophotocoagulation versus trabeculectomy. Middle East African J Ophthalmol. 2017;24(4):177-182. doi: 10.4103/meajo.MEAJO_232_16

8. Choy BNK. Comparison of surgical outcome of trabeculectomy and phacotrabeculectomy in Chinese glaucoma patients. Int J Ophthalmol. 2017;10(12):1928-1930. doi: 10.18240/ijo.2017.12.23

9. Song BJ, Ramanathan M, Morales E, Law SK, Giaconi JA, Coleman AL, Caprioli J. Trabeculectomy and combined phacoemulsification-trabeculectomy: outcomes and risk factors for failure in primary angle closure glaucoma. J Glaucoma. 2016;25(9):763-769. doi: 10.1097/ IJG.0000000000000493

10. Qiu HY, Chen JK, Yu Z. Application of intraoperative adjustable suture in trabeculectomy. Chin J Pract Ophthalmol. 2014;32(3):341-342. doi: 10.3760/cma.j.is sn.1006-4443.2014.03.022

11. Lei JC. Observation of mitomycin in combination with trabeculectomy and adjustable sutured scleral flap. Chin J Mod Drug Appl. 2016;10(12):233-234. doi: 10.14164/j.cnki. cn11-5581/r.2016.12.176

12. Tian CL. Sclerai lamellar draining pool type trabeculectomy for refractory glaucoma. J Injuries Occupat Dis Eye Ophthalmic Surg. 2010;32(7):541-543

13. Ge J, Sun $\mathrm{XH}$, Wang NL. Research Progress of Modern Glaucoma. Beijing: Science Press. 2000;164-169.

14. Liu ML, Wang YX. The prevalence of blindness caused by primary angle closure glaucoma in middle-aged Chinese population: a systematic review and meta-analysis. Chin J Ophthalmol. 2017;53(5). doi: 10.3760/cma.j.is sn.0412-4081.2017.05.010

15. Seet LF, Narayanaswamy A, Finger SN, Htoon HM, Nongpiur ME, Toh LZ, et al. Distinct iris gene expression profiles of primary angle closure glaucoma and primary open angle glaucoma and their interaction with ocular biometric parameters. Clin Exp Ophthalmol. 2016;44(8):684692. doi: $10.1111 /$ ceo.12743
16. Actis AG, Versino E, Brogliatti B, Rolle T. Risk factors for primary open angle glaucoma (poag) progression: A study ruled in torino. Open Ophthalmol J. 2016;10:129-139. doi: 10.2174/1874364101610010129.

17. Zhang $\mathrm{XL}$, Huang WB. Is increased choroidal thickness a risk factor for primary angle-closure glaucoma? Chin J Ophthalmol. 2015;51(2):86-88. doi: 10.3760/cma.j.is sn.0412-4081.2015.02.003

18. Serna-Ojeda JC, Guadarrama-Vallejo DC, Valencia-Aguirre JD, Cordova-Cervantes J, Matiz-Moreno H, Korder-Ortega V. Glaucoma maligno en paciente con cirugia previa de catarata traumatica e implante valvular: reporte de caso. Rev Mex Oftalmol. 2015;89(3):179-182. doi: 10.1016/j. mexoft.2015.01.001

19. Liu JH, Lin HY, Tzeng SH, Chao SC. Comparison of trabeculectomy with Ex-PRESS shunt implantation in primary-open-angle-glaucoma patients: A retrospective study. Taiwan J Ophthalmol. 2015;5(3):120-123. doi: 10.1016/j.tjo.2015.04.004

20. Mohamed TH, Salman AG, Elshinawy RF. Trabeculectomy with Ologen implant versus mitomycin $C$ in congenital glaucoma secondary to Sturge Weber Syndrome. Int J Ophthalmol. 2018;11(2):251-255. doi: 10.18240/ijo.2018.02.12

21. Bussel II, Kaplowitz K, Schuman JS, Loewen NA. Trabectome Study Group. Outcomes of ab interno trabeculectomy with the trabectome after failed trabeculectomy. BritJOphthalmol. 2015;99(2):258-262. doi: 10.1136/bjophthalmol-2013-304717

22. Castejon MA, Teus MA, Bolivar G, Paz-MorenoArrones J, Castano B. Outcomes of trabeculectomy and phacotrabeculectomy with collagen matrix implant (ologen) and low-dose mitomycin C: 2-year follow-up. J Glaucoma. 2018;27(1):50-54.

23. Xia SG, Xiao QG, Peng HC. Curative effect observation on modified trabeculectomy combined with interscleral drainage pond forming for acute angle-closure glaucoma. Int J Ophthalmol. 2011;11(7):1210-1211. doi: 10.3969/j. issn.1672-5123.2011.07.026

\section{Authors' Contribution:}

LXH \& SSY: Study design, data collection and analysis.

CLL, WHZ \& SSY: Manuscript preparation, drafting and revising.

LXH \& SSY: Review, final approval of manuscript, are responsible for integrity of research.

Authors:

1. Li Xiahou

2. Chunlan Liu

3. Weihong Zhou

4. Shasha Yang

The Second Affiliated Hospital,

South China University of Technology,

Guangzhou First People's Hospital,

Guangzhou, 510180, China.

1-3: Department of Ophthalmology,

Xinyu People's Hospital,

Xinyu, Jiangxi Province, 338000, China. 